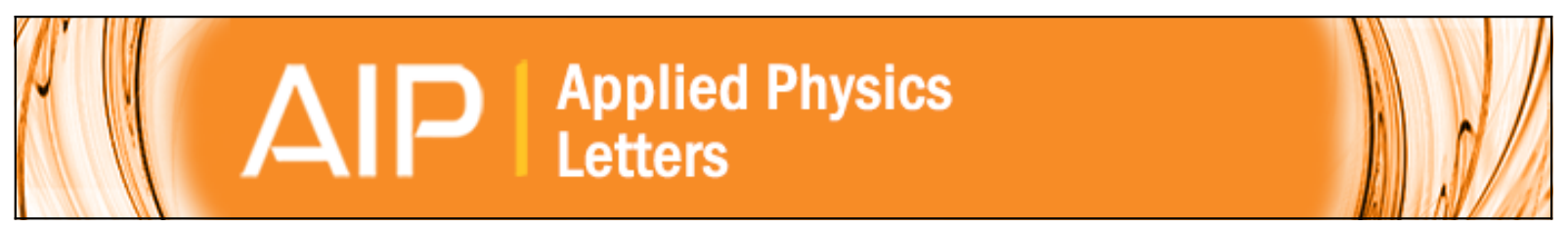

\title{
Carrier and heat transport properties of polycrystalline GeSn films on SiO2
}

Noriyuki Uchida, Tatsuro Maeda, Ruben R. Lieten, Shingo Okajima, Yuji Ohishi, Ryohei Takase, Manabu Ishimaru, and Jean-Pierre Locquet

Citation: Applied Physics Letters 107, 232105 (2015); doi: 10.1063/1.4937386

View online: http://dx.doi.org/10.1063/1.4937386

View Table of Contents: http://scitation.aip.org/content/aip/journal/apl/107/23?ver=pdfcov

Published by the AIP Publishing

\section{Articles you may be interested in}

Manipulation of polycrystalline $\mathrm{TiO} 2$ carrier concentration via electrically active native defects

J. Vac. Sci. Technol. A 29, 061503 (2011); 10.1116/1.3635373

Molecular orbital ordering in titania and the associated semiconducting behavior Appl. Phys. Lett. 99, 142104 (2011); 10.1063/1.3646105

Evaluation of microstructures and carrier transport behaviors during the transition process from amorphous to nanocrystalline silicon thin films

J. Appl. Phys. 105, 054901 (2009); 10.1063/1.3087500

Structural and electrical properties of Ge $1 \mathrm{Sb} 2 \mathrm{Te} 4$ face centered cubic phase

J. Appl. Phys. 104, 103712 (2008); 10.1063/1.3021462

Properties of crystallized Si $1-x$ Ge $x$ thin films deposited by sputtering

J. Vac. Sci. Technol. A 15, 2836 (1997); 10.1116/1.580836

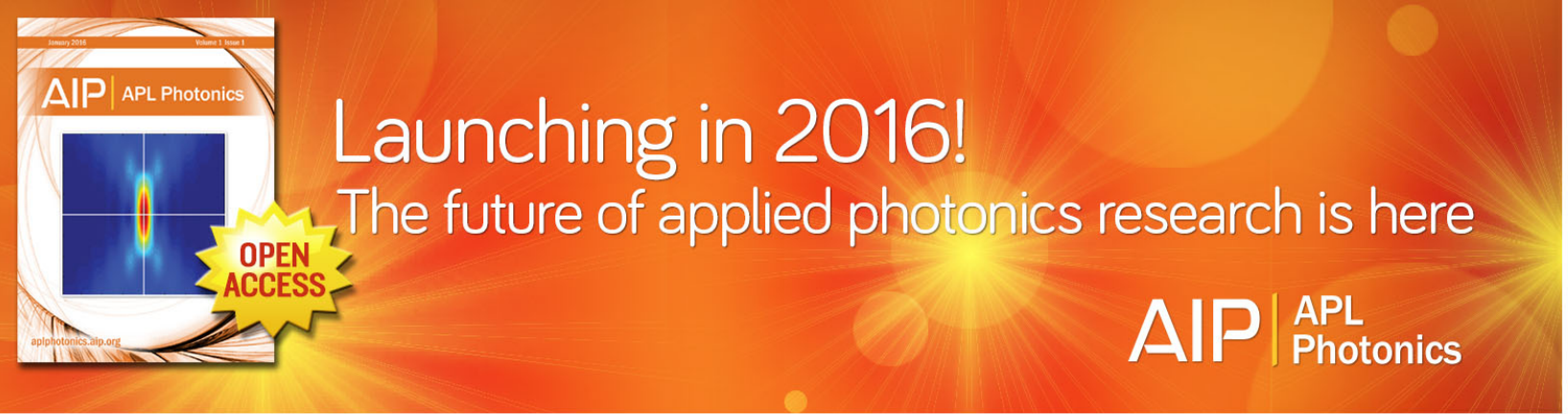




\title{
Carrier and heat transport properties of polycrystalline $\mathrm{GeSn}$ films on $\mathrm{SiO}_{2}$
}

\author{
Noriyuki Uchida, ${ }^{1}$ Tatsuro Maeda, ${ }^{1}$ Ruben R. Lieten, ${ }^{2,3}$ Shingo Okajima, ${ }^{4}$ Yuji Ohishi, ${ }^{4}$ \\ Ryohei Takase, ${ }^{5}$ Manabu Ishimaru, ${ }^{5}$ and Jean-Pierre Locquet ${ }^{3}$ \\ ${ }^{1}$ Nanoelectronics Research Institute, Advance Industrial Science and Technology, Central 4, 1-1-1 Higashi, \\ Tsukuba, Ibaraki 305-8562, Japan \\ ${ }^{2}$ IMEC, 3001 Leuven, Belgium \\ ${ }^{3}$ Department of Physics and Astronomy, KU Leuven, 3001 Leuven, Belgium \\ ${ }^{4}$ Graduate School of Engineering, Osaka University, 2-1 Yamadaoka, Suita, Osaka 565-0871, Japan \\ ${ }^{5}$ Department of Materials Science and Engineering, Kyushu Institute of Technology, Tobata, Kitakyushu, \\ Fukuoka 804-8550, Japan
}

(Received 14 July 2015; accepted 22 November 2015; published online 10 December 2015)

\begin{abstract}
We evaluated the potential of polycrystalline (poly-) GeSn as channel material for the fabrication of thin film transistors (TFTs) at a low thermal budget $\left(<600^{\circ} \mathrm{C}\right)$. Poly-GeSn films with a grain size of $\sim 50 \mathrm{~nm}$ showed a carrier mobility of $\sim 30 \mathrm{~cm}^{2} \mathrm{~V}^{-1} \mathrm{~s}^{-1}$ after low-temperature annealing at $475-500{ }^{\circ} \mathrm{C}$. Not only carrier mobility but also thermal conductivity of the films is important in assessing the self-heating effect of the poly-GeSn channel TFT. The thermal conductivity of the poly-GeSn films is $5-9 \mathrm{~W} \mathrm{~m}^{-1} \mathrm{~K}^{-1}$, which is significantly lower compared with $30-60 \mathrm{~W} \mathrm{~m}^{-1}$ $\mathrm{K}^{-1}$ of bulk Ge; this difference results from phonon scattering at grain boundaries and Sn interstitials. The poly-GeSn films have higher carrier mobility and thermal conductivity than poly-Ge films annealed at $600{ }^{\circ} \mathrm{C}$, because of the improved crystal quality and coarsened grain size resulting from Sn-induced crystallization. Therefore, the poly-GeSn film is well-suited as channel material for TFTs, fabricated with a low thermal budget. (C) 2015 AIP Publishing LLC.
\end{abstract}

[http://dx.doi.org/10.1063/1.4937386]

Owing to high carrier mobility, the polycrystalline GeSn (poly-GeSn) film constitutes a suitable channel material for high-performance thin film transistors (TFTs). Its mobility is higher than the mobilities of $\mathrm{Si}$ and $\mathrm{Ge}$ individually., Moreover, the thermal budget required to fabricate a TFT can be reduced using poly-GeSn film as the channel material, because of its low eutectic temperature $\left(231.1^{\circ} \mathrm{C}\right)$ and correspondingly low crystallization temperature. ${ }^{3-6}$ Poly-GeSn films have, therefore, significant potential for the use in TFTs. However, poly-Si TFTs on glass substrates are susceptible to self-heating. ${ }^{7}$ The heat generated during the TFT operation is localized near the drain edge of the structures because of the low thermal conductivity of the substrates. Furthermore, the temperature is distributed inhomogeneously in the channel region, thereby suppressing the TFT driving current and the threshold voltage shift in current-voltage curves. $^{7}$

Self-heating is detrimental to the poly-Ge based TFT channel materials because of the lower thermal conductivity of Ge compared with that of Si. Indeed, while poly-Si and bulk Si have similar high thermal conductivities $(60-140 \mathrm{~W}$ $\left.\mathrm{m}^{-1} \mathrm{~K}^{-1}\right)^{8}$ that of bulk Ge that is only $50-60 \mathrm{~W} \mathrm{~m}^{-1} \mathrm{~K}^{-1}$ depending on carrier concentration. ${ }^{9}$ Furthermore, high levels of phonon scattering at grain boundaries in polycrystalline structures reduce thermal conductivity. ${ }^{8}$ With the formation of nano-sized grains in poly-Si, this reduction is up to $90 \% .^{8}$ Moreover, GeSn alloying induces additional phonon scattering, similar to SiGe alloying. ${ }^{10}$ Therefore, GeSn has a significantly lower thermal conductivity than that of bulk Ge because of these grain boundary and alloy scattering effects. The thermal conductivity of poly-GeSn is also expected to be lower than that of poly-Si. To evaluate the potential of poly-GeSn for the use as a TFT channel, the carrier and heat transport properties must be simultaneously investigated.

In this work, we investigated the electrical (carrier transport) and thermal properties (thermal conductivity $\kappa$ ) of GeSn layers deposited on $\mathrm{SiO}_{2} / \mathrm{Si}$ substrates. The GeSn films are deposited with an amorphous structure and are transformed into poly-crystalline GeSn (poly-GeSn) during thermal annealing. Additionally, the relation between phonon scattering and the $\kappa$ of the poly-GeSn films was elucidated by numerically simulating $\kappa$ using a frequency-dependent model based on the Debye dispersion. ${ }^{8}$

We deposited a-GeSn on $\mathrm{SiO}_{2} / \mathrm{Si}$ using molecular beam deposition. After ultrasonically cleaning the Si substrate in acetone, a $1-\mu$ m-thick $\mathrm{SiO}_{2}$ layer was sputter-deposited using a $\mathrm{Si}$ target in $\mathrm{Ar}$ and $\mathrm{O}_{2}$ gas. The a-GeSn film was deposited on this $\mathrm{SiO}_{2}$ layer at $\sim 10^{\circ} \mathrm{C}$ using a similar method described in the literature. ${ }^{2}$ Ge $(99.99 \%)$ and $\mathrm{Sn}(99.99 \%)$ were evaporated from separate effusion cells in an ultrahigh vacuum chamber at a base pressure of $2.6 \times 10^{-8} \mathrm{~Pa}$; molecular beam pressures of $2.1 \times 10^{-5} \mathrm{~Pa}$ and $3.6 \times 10^{-6} \mathrm{~Pa}$ were used for $\mathrm{Ge}$ and $\mathrm{Sn}$, respectively. The thickness of all aGeSn films used was $100 \mathrm{~nm}$. The concentration of $\mathrm{Sn}$ in the a-GeSn film was estimated as $13.6 \pm 0.3 \%$ via Rutherford back-scattering (RBS) measurements using a ${ }^{4} \mathrm{He}^{2+}$ ion beam operating at $2300 \mathrm{keV}$. For comparison, we also deposited an amorphous non-Sn-doped Ge (i.e., a-Ge) film on $\mathrm{SiO}_{2} / \mathrm{Si}$ substrates using the same molecular beam method. The a-GeSn and a-Ge films were annealed via halogen lamp heating at $350-600{ }^{\circ} \mathrm{C}$ in $\mathrm{N}_{2}$ gas for $30 \mathrm{~min}$. Cross-sectional transmission electron microscope (TEM) images of the GeSn films were obtained at an electron beam energy of 
$200 \mathrm{kV}$, before and after annealing. Additionally, the GeSn films were characterized by X-ray diffraction (XRD) using Co K-alpha $(\lambda=0.1789 \mathrm{~nm})$ radiation. Raman spectra were also obtained from the films; 488-nm-wavelength lasers with a power of $0.14 \mathrm{~mW}$ were used to excite the $\sim 2-\mu \mathrm{m}$-diameter samples under a microscope objective of $\times 50$. Hall measurements performed at $300 \mathrm{~K}$ characterized the electrical transport properties of the GeSn films. Moreover, the $\kappa$ values of the films were measured at $300 \mathrm{~K}$ via a thermoreflectance method, using a thin film thermal conductivity meter (TCN-2 $\omega$, ULVAC-RIKO Corp.). ${ }^{11}$

Figure 1 shows the structural evolution of the GeSn film during the thermal annealing process. The cross-sectional bright-field TEM images and selected-area electron diffraction (SAED) patterns [Fig. 1(a)] reveal that the as-deposited specimen has an amorphous structure. However, the film starts to crystallize after annealing at $350^{\circ} \mathrm{C}$ [Fig. 1(b)], as evidenced by the appearance of nanocrystallites (NCs) and weak spots with halo rings in the SAED pattern; these NCs have diameters of $5-10 \mathrm{~nm}$. Amorphous Ge has a crystallization temperature of $\sim 600^{\circ} \mathrm{C}$ on the $\mathrm{SiO}_{2}$ layer; therefore, the
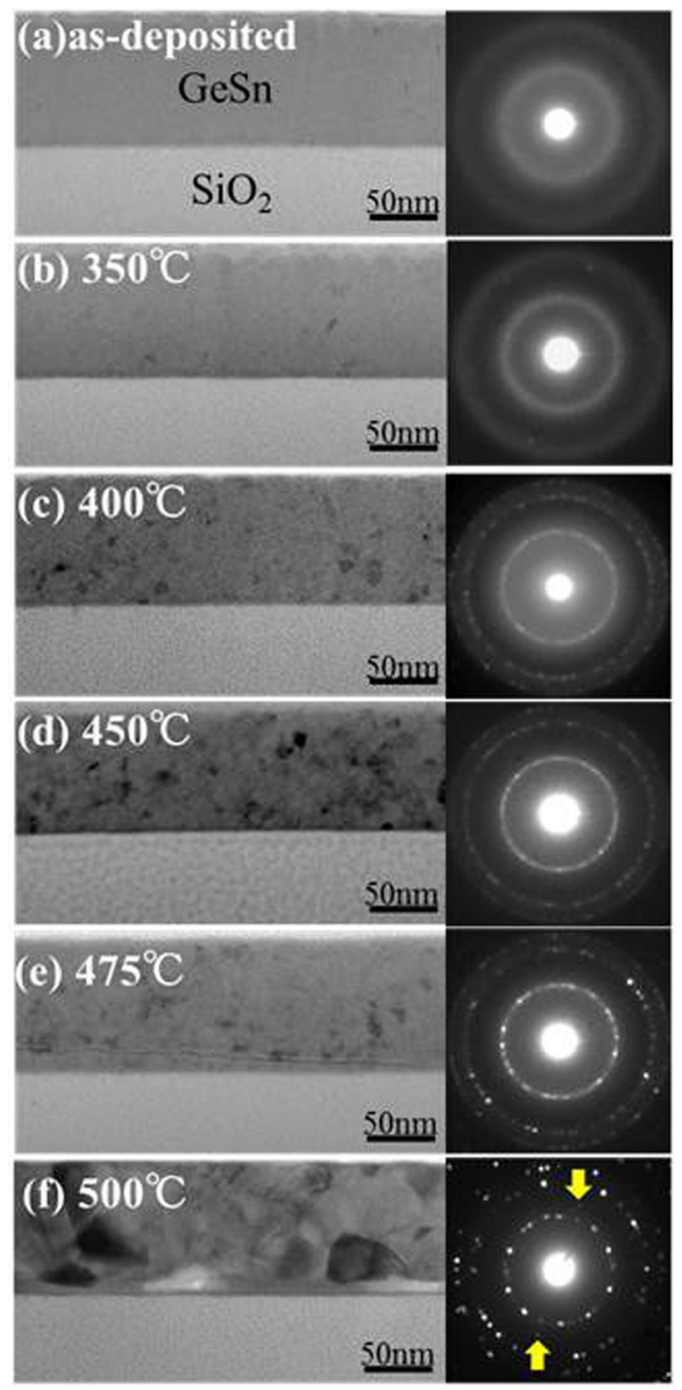

FIG. 1. Cross-sectional bright-field TEM images and SAED patterns of the GeSn films (a) before and after annealing at (b) $350{ }^{\circ} \mathrm{C}$, (c) $400{ }^{\circ} \mathrm{C}$, (d) $450^{\circ} \mathrm{C}$, (e) $475^{\circ} \mathrm{C}$, and (f) $500^{\circ} \mathrm{C}$. The arrows indicate the diffraction spots corresponding to the $\mathrm{Sn}$ crystal. lower crystallization temperature of the GeSn film is attributed to Sn doping, as reported in the literature. ${ }^{3,4}$ Further annealing enhances the formation of high density NCs [Fig. 1(c)], for which sizes increase with increasing annealing temperature. Additionally, at temperatures higher than $500{ }^{\circ} \mathrm{C}$, typical polyhedral-shaped $\mathrm{NCs}$ form throughout the films, indicating the disappearance of an amorphous region in GeSn films. Bright spots (marked with arrows) between the 111 and 220 rings of the Ge crystals in the SAED pattern [Fig. 1(f)] also result from the formation of Sn crystals, indicating the Sn segregation.

The NC diameters of $3.5-22 \mathrm{~nm}, 5.0-24 \mathrm{~nm}, 9.0-27 \mathrm{~nm}$, and $24-91 \mathrm{~nm}$ were derived from TEM images [Figs. $1(\mathrm{c})-1(\mathrm{f})]$ annealed at $400^{\circ} \mathrm{C}, 450^{\circ} \mathrm{C}, 475^{\circ} \mathrm{C}$, and $500^{\circ} \mathrm{C}$, respectively; these NCs had corresponding average sizes of $10 \mathrm{~nm}, 13 \mathrm{~nm}, 15 \mathrm{~nm}$, and $47 \mathrm{~nm}$. The significant increase of $\mathrm{NC}$ sizes in the $500^{\circ} \mathrm{C}$-annealed specimen is attributed to the Sn-induced crystallization of the films.

Figure 2 shows the XRD $2 \theta$-scan profiles obtained at various annealing temperatures of the films. Bragg peaks corresponding to GeSn NCs with Miller indices of (111), (220), and (311) are clearly observed. The position of each peak shifts to a higher scattering angle; the peaks become narrow with increasing temperature. These changes are attributed to the increased crystal quality of the NCs and the decreased Sn concentration. We can estimate the lattice constants of the GeSn NCs (Table I) from the positions of the (111) peak. The concentration of Sn in the NCs is determined from the lattice constant using Vegard's law, assuming that the GeSn layer is not strained. The $450^{\circ} \mathrm{C}$-annealed film had an estimated Sn concentration of $14 \%$, which concurred with the RBS result, suggesting that the assumption of limited lattice strain was reasonable. The concentration of $\mathrm{Sn}$ in the GeSn lattice decreased with increasing annealing temperature of $470{ }^{\circ} \mathrm{C}$ and $500{ }^{\circ} \mathrm{C}$, indicating that Sn segregations occurred during annealing.

We examined the structural changes from amorphous to polycrystalline by measuring the Raman spectra [Fig. 3(a)] of the films annealed at $400{ }^{\circ} \mathrm{C}, 470{ }^{\circ} \mathrm{C}$, and $500{ }^{\circ} \mathrm{C}$. The

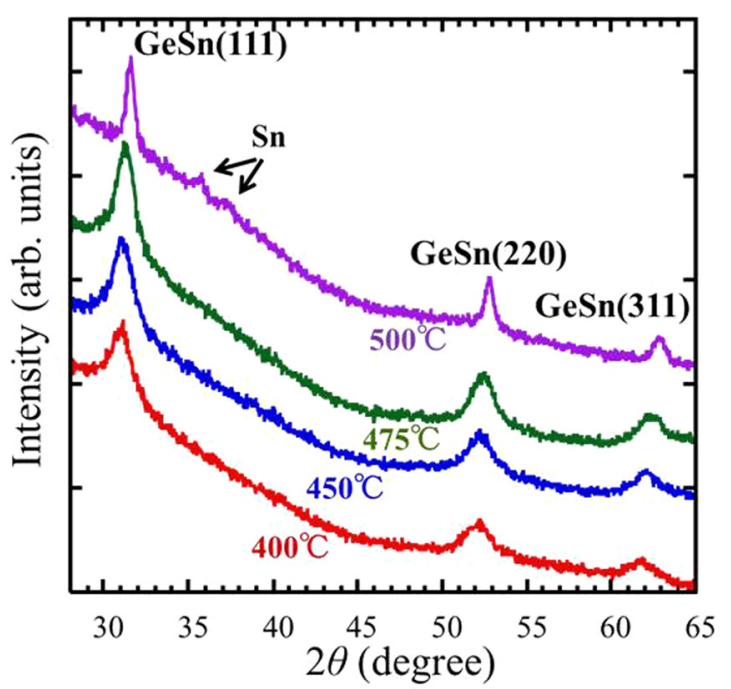

FIG. 2. XRD 2 $\theta$-scan profiles of GeSn films annealed at temperatures of $400-500^{\circ} \mathrm{C}$. 
TABLE I. Dependence on annealing temperature $\left(T_{\mathrm{a}}\right)$ of lattice constants $\left(L_{\mathrm{Ge}}\right)$ of $\mathrm{Ge}$, as determined from XRD spectra, $\mathrm{Ge}_{1-x} \mathrm{Sn}_{x}$ concentration $(x)$, calculated using Vegard's law. Given bulk Ge and Sn lattice spacings of $0.566 \mathrm{~nm}$ and $0.649 \mathrm{~nm}$, respectively, the $x$ values are obtained from $L_{\mathrm{Ge}}=0.566 \times(1-x)+0.649 \times x$.

\begin{tabular}{lcc}
\hline \hline Ta $\left({ }^{\circ} \mathrm{C}\right)$ & $L_{\mathrm{Ge}}(\mathrm{nm})$ & $x$ \\
\hline 400 & 0.579 & $0.16(16 \%)$ \\
450 & 0.577 & $0.14(14 \%)$ \\
475 & 0.574 & $0.1(10 \%)$ \\
500 & 0.570 & $0.05(5 \%)$ \\
\hline \hline
\end{tabular}

phonon bands corresponding to Ge occur at Raman shifts of $285 \mathrm{~cm}^{-1}-296 \mathrm{~cm}^{-1}$ in the spectra. The low-frequency tail of the band corresponding to the $400^{\circ} \mathrm{C}$-annealed films results from the amorphous regions of the films. The amorphous GeSn layer typically gives rise to a broad peak at $\sim 260 \mathrm{~cm}^{-1}$ in the Raman spectra. ${ }^{2}$ The peak position shifts to a higher frequency [Fig. 3(b)] and approaches that of bulk Ge $\left(300 \mathrm{~cm}^{-1}\right)$ with increasing annealing temperature. This shift stems from decreases in the concentration of $\mathrm{Sn}$ in the NCs with increasing temperature (Table I), as reported by Lin et al. ${ }^{12}$ for single crystal of epitaxially grown GeSn. The SAED pattern and XRD spectrum of GeSn films annealed at $500{ }^{\circ} \mathrm{C}$ show $\mathrm{Sn}$ segregation in the film. We found the $\mathrm{Sn}$
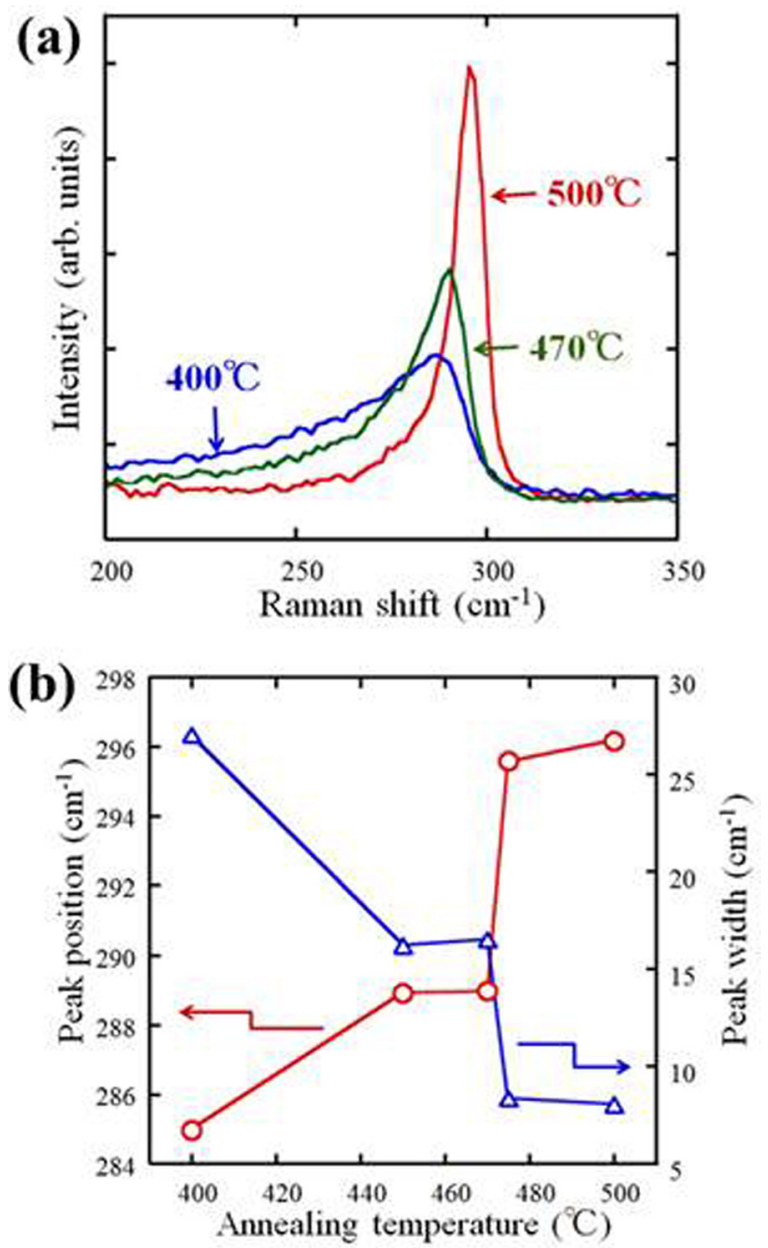

FIG. 3. (a) Raman spectra of the GeSn films annealed at $400^{\circ} \mathrm{C}, 470{ }^{\circ} \mathrm{C}$, and $500^{\circ} \mathrm{C}$. (b) The peak frequency and FWHM of Ge phonons near $\sim 300 \mathrm{~cm}^{-1}$ in the films annealed at $400-500^{\circ} \mathrm{C}$. segregation and/or precipitation of $\mathrm{Sn}$ at the interface between the GeSn and $\mathrm{SiO}_{2}$ layer in high-angle annular dark-field scanning TEM (HAADF-STEM) images of $475^{\circ} \mathrm{C}$ and $500^{\circ} \mathrm{C}$ samples. Typical sizes of $\mathrm{Sn}$ NCs ranged from $5-10 \mathrm{~nm}$ and $50-70 \mathrm{~nm}$ in diameter for the $475^{\circ} \mathrm{C}$ and $500{ }^{\circ} \mathrm{C}$ samples, respectively.

The full-width at half maximum (FWHM) of the Ge phonons of the films decreased from $17 \mathrm{~cm}^{-1}$ at an annealing temperature of $475^{\circ} \mathrm{C}$ to $8 \mathrm{~cm}^{-1}$ at $470^{\circ} \mathrm{C}$. This narrowing of the phonon-related peaks indicates that the crystal quality of the film increases with increasing annealing temperature. The carrier mobility also increased at temperatures higher than $475^{\circ} \mathrm{C}$. Indeed, a relatively small increase of $5^{\circ} \mathrm{C}$ in annealing temperature from $470{ }^{\circ} \mathrm{C}$ to from $475^{\circ} \mathrm{C}$ had a significant effect on the ability to improve the crystal quality of GeSn lattice. The improvement of crystal quality occurs at the same time as the segregation of Sn NCs in the films.

Figure 4 shows the dependence of the Hall carrier mobility and concentration of the poly-GeSn films on annealing temperatures of $400-500^{\circ} \mathrm{C}$; densities of $\sim 10^{19} \mathrm{~cm}^{-3}$ are consistent with that of a p-type hole carrier semiconductor. Additionally, the films annealed at temperatures lower than $470{ }^{\circ} \mathrm{C}$, and at $475-500{ }^{\circ} \mathrm{C}$ exhibited mobilities of $1-5 \mathrm{~cm}^{2}$ $\mathrm{V}^{-1} \mathrm{~s}^{-1}$ and $27-30 \mathrm{~cm}^{2} \mathrm{~V}^{-1} \mathrm{~s}^{-1}$, respectively. This increase in carrier mobility stems from the improved crystal quality; i.e., annealing resulted in a decrease in the defect density of the film. Moreover, the decrease in the carrier density from $5.6 \times 10^{19} \mathrm{~cm}^{-3}$ to $1.2 \times 10^{19} \mathrm{~cm}^{-3}$ is attributed to a reduction in the density of acceptor-like defects during annealing. The estimated hole mobility and density of the poly-Ge film were $12 \mathrm{~cm}^{2} \mathrm{~V}^{-1} \mathrm{~s}^{-1}$ and $2.2 \times 10^{18} \mathrm{~cm}^{-3}$, respectively, even after annealing at temperatures higher than $600{ }^{\circ} \mathrm{C}$. High hole mobility and density render GeSn films wellsuited for use in low-thermal-budget TFTs.

The thermal conductivity values $(\kappa)$ of GeSn films were determined at $300 \mathrm{~K}$ via a thermo-reflectance $2 \omega$ method. ${ }^{11}$ The measured sample consisted of four layers: an $\mathrm{Au}$ thin film $(100 \mathrm{~nm})$, the GeSn or Ge film $(100 \mathrm{~nm})$, the $\mathrm{SiO}_{2}$ film $(1 \mu \mathrm{m})$, and the Si substrate. The Au film was deposited onto the GeSn or Ge film by DC magnetron sputtering of the Au

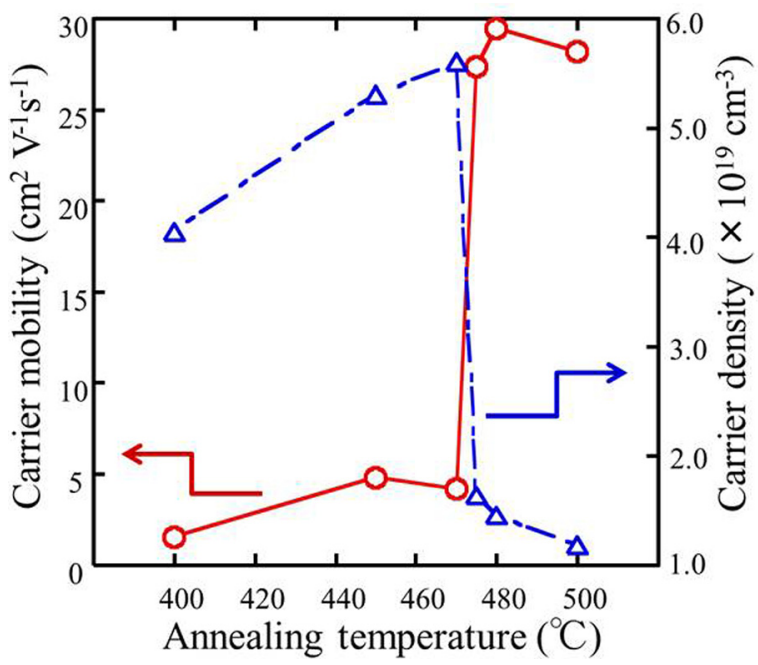

FIG. 4. Carrier mobility and concentration of poly-GeSn films annealed at temperatures of $400-500{ }^{\circ} \mathrm{C}$. 
target. This film was used as an AC Joule heating source and a sensor for detecting the temperature response via the thermo-reflectance technique. For the current sample structure, $\kappa$ values of up to $5 \mathrm{~W} \mathrm{~m}^{-1} \mathrm{~K}^{-1}$ can be measured using this $2 \omega$ method. Figure 5 shows the dependence of the $\kappa$ of the GeSn and Ge films on the annealing temperature. Prior to annealing, both films exhibited a similar $\kappa\left(\sim 1 \mathrm{~W} \mathrm{~m}^{-1} \mathrm{~K}^{-1}\right)$ to that of amorphous $\mathrm{Ge}(\mathrm{a}-\mathrm{Ge}){ }^{13}$ The $\kappa$ of both films also increased with increasing annealing temperature. However, the GeSn films annealed at $300-350^{\circ} \mathrm{C}$, which consisted predominantly of amorphous regions [Fig. 1(b)], had a slightly higher $\kappa\left(2.1-2.3 \mathrm{~W} \mathrm{~m}^{-1} \mathrm{~K}^{-1}\right)$ than the a-Ge film. This suggests that the short-range ordering of the Ge networks improved with annealing, as evidenced by the formation of nanocrystals in the $350{ }^{\circ} \mathrm{C}$-annealed film [Fig. 1(b)]. The $\kappa$ increased further owing to poly-crystallization of the films at temperatures higher than $400^{\circ} \mathrm{C}$. The films annealed at $400^{\circ} \mathrm{C}$ and $450-470{ }^{\circ} \mathrm{C}$ had $\kappa$ values of $2.7 \mathrm{~W} \mathrm{~m}^{-1} \mathrm{~K}^{-1}$ and 4.6-4.7 $\mathrm{W} \mathrm{m}^{-1} \mathrm{~K}^{-1}$, respectively. In contrast, the $\kappa$ of bulk Ge varies from $30-60 \mathrm{~W} \mathrm{~m}^{-1} \mathrm{~K}^{-1}$, as mentioned above. ${ }^{9}$ The significantly lower $\kappa$ is attributed to enhanced phonon scattering by $\mathrm{Sn}$ interstitials and grain boundaries in the poly-GeSn films. The $\kappa$ values obtained concur with those calculated for Ge [Fig. 5] using a similar calculation method in the literature, ${ }^{14}$ based on the models of Klemens ${ }^{15}$ and Callaway. ${ }^{16}$ In the calculation, we assumed that phonon scattering stemmed from domain boundary scattering, phononphonon (Umklapp) scattering; parameters and functional forms are provided in the supplementary material. ${ }^{17-19}$ For the calculations of the domain boundary scattering, we used the average size of the NCs, the domain size, and the concentrations of Sn listed in Table I; the average size of the NCs was determined from TEM images (Fig. 1). The calculated $\kappa$ values of $\mathrm{GeSn}$ films, which were annealed at $400^{\circ} \mathrm{C}$, $450{ }^{\circ} \mathrm{C}$, and $475^{\circ} \mathrm{C}$, were close to the $\kappa$ values estimated by the $2 \omega$ method, suggesting that the calculation model provides reasonable estimates of the $\kappa$ values. The $2 \omega$ method was not used to estimate the $\kappa$ of the samples annealed at temperatures higher than $500^{\circ} \mathrm{C}$, because of the detection

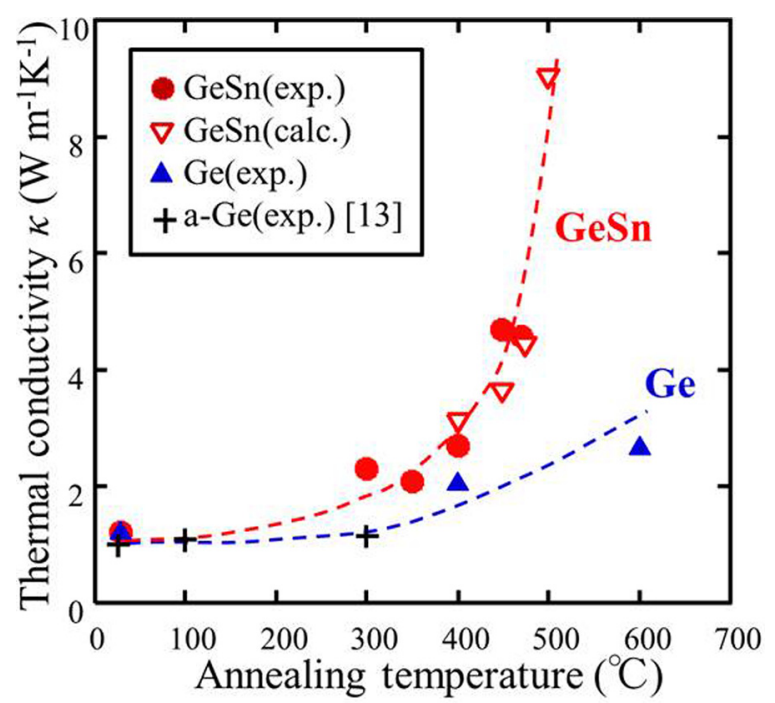

FIG. 5. Thermal conductivity of GeSn and Ge films before and after annealing at $300-600^{\circ} \mathrm{C}$. limit of $5 \mathrm{~W} \mathrm{~m}^{-1} \mathrm{~K}^{-1}$, as mentioned above. Indeed, the thermal conductivity of the $500^{\circ} \mathrm{C}$-annealed sample was calculated to be $9.0 \mathrm{~W} \mathrm{~m} \mathrm{~m}^{-1} \mathrm{~K}^{-1}$, obtained by numerical calculation [Fig. 5]. The $\kappa\left(2.3 \mathrm{~W} \mathrm{~m}^{-1} \mathrm{~K}^{-1}\right)$ of the poly-Ge sample annealed at $600^{\circ} \mathrm{C}$, was, however, significantly lower than those of the poly-GeSn films annealed at $475^{\circ} \mathrm{C}-500^{\circ} \mathrm{C}$. This lower $\kappa$ resulted from the improved crystal quality and coarsened grain size because of Sninduced crystallization. The poly-GeSn films have, therefore, higher $\kappa$ values than their poly-Ge counterparts during lowtemperature $\left(<500^{\circ} \mathrm{C}\right)$ annealing. This indicates that when TFT is fabricated at a low thermal budget, the poly-GeSn film should exhibit better carrier and thermal transport properties for the channel material than the poly-Ge film.

Here, we benchmark the poly-GeSn film for TFT channel materials. The poly-GeSn film has an advantage with lower thermal budget than poly-Si films. However, measures must be taken to prevent or at least reduce self-heating of the poly-GeSn-channel TFT; this heating results from the lower $\kappa$ of poly-GeSn $\left(5-9 \mathrm{~W} \mathrm{~m}^{-1} \mathrm{~K}^{-1}\right)$ than poly-Si $(60-140 \mathrm{~W}$ $\left.\mathrm{m}^{-1} \mathrm{~K}^{-1}\right){ }^{8}$ The amorphous $\mathrm{Si}$ (a-Si) films, amorphous InGaZnO (a-IGZO) films, and films of organic semiconductors, for example, pentacene, are also useful as TFT channels fabricated at low thermal budgets. However, the $\kappa$ of these films is estimated to be $1-2 \mathrm{~W} \mathrm{~m}^{-1} \mathrm{~K}^{-1}$ for a-Si film, ${ }^{20}$ $1.4 \mathrm{~W} \mathrm{~m}^{-1} \mathrm{~K}^{-1}$ for a-IGZO film, ${ }^{21}$ and $0.51 \mathrm{~W} \mathrm{~m}^{-1} \mathrm{~K}^{-1}$ for pentacene film ${ }^{22}$ lower than poly-GeSn films because of the amorphous structures. Thus, the poly-GeSn films have an advantage over these films in suppressing heat from selfheating of the TFT channel.

In conclusion, poly-GeSn films $(\mathrm{Sn}=13.6 \%)$ were synthesized on $\mathrm{SiO}_{2} / \mathrm{Si}$ substrates by thermal annealing at $400-500^{\circ} \mathrm{C}$ in $\mathrm{N}_{2}$ gas. The GeSn NCs had average grain sizes of up to $\sim 50 \mathrm{~nm}$ after annealing at $500^{\circ} \mathrm{C}$. Additionally, the hole carrier mobility and $\kappa$ of the GeSn films increased to maximum values of $30 \mathrm{~cm}^{2} \mathrm{~V}^{-1} \mathrm{~s}^{-1}$ and $9.0 \mathrm{~W} \mathrm{~m}^{-1} \mathrm{~K}^{-1}$, respectively, with annealing. The films also had higher hole mobility and $\kappa$ than their Ge counterparts, when annealed at temperatures lower than $500^{\circ} \mathrm{C}$; this resulted from an improved crystal quality and increased grain size because of Sn-induced crystallization. The results indicate that the poly-GeSn film is well-suited as channel material for TFTs, fabricated with a low thermal budget.

This work was supported by the Bilateral Joint Research Projects and MEXT KAKENHI Grant No. $25289220 / J S P S$, the $A L C A / J S T$, and a cooperative program (Proposal No.15G000) of the CRDAM-IMR, Tohoku University.

${ }^{1}$ J. D. Sau and M. L. Cohen, Phys. Rev. B 75, 045208 (2007).

${ }^{2}$ R. R. Lieten, T. Maeda, W. Jevasuwan, H. Hattori, N. Uchida, S. Miura, M. Tanaka, and J. P. Locquet, Appl. Phys. Express 6, 101301 (2013).

${ }^{3}$ O. Nakatsuka, K. Mochizuki, Y. Shimura, T. Yamaha, and S. Zaima, Thin Solid Films 520, 3288 (2012).

${ }^{4}$ M. Kurosawa, N. Taoka, M. Sakashita, O. Nakatsuka, M. Miyao, and S. Zaima, Appl. Phys. Lett. 103, 101904 (2013).

${ }^{5}$ K. Toko, N. Oya, N. Saitoh, N. Yoshizawa, and T. Suematsu, Appl. Phys. Lett. 106, 082109 (2015).

${ }^{6}$ W. Takeuchi, N. Taoka, M. Kurosawa, M. Sakashita, O. Nakatsuka, and S. Zaima, Appl. Phys. Lett. 107, 022103 (2015). 
${ }^{7}$ T. Sameshima, Y. Sunaga, and A. Kohno, Jpn. J. Appl. Phys., Part 2 35, L308 (1996).

${ }^{8}$ Z. Wang, J. E. Alaniz, W. Jang, J. E. Garay, and C. Dames, Nano Lett. 11, 2206 (2011).

${ }^{9}$ B. Abeles, J. Phys. Chem. Solids 8, 340 (1959).

${ }^{10}$ E. F. Steigmeier and B. Abeles, Phys. Rev. 136, A1149 (1964).

${ }^{11}$ R. Kato and I. Hatta, Int. J. Thermophys. 26, 179 (2005).

${ }^{12}$ H. Lin, R. Chen, Y. Huo, T. I. Kamins, and J. S. Harris, Appl. Phys. Lett. 98, 261917 (2011).

${ }^{13}$ T. Zhan, Y. Xu, M. Goto, Y. Tanaka, R. Kato, M. Sasaki, and Y. Kagawa, Appl. Phys. Lett. 104, 071911 (2014).

${ }^{14}$ D. T. Morelli, J. P. Heremans, and G. A. Slack, Phys. Rev. B 66, 195304 (2002).
${ }^{15}$ P. G. Klemens, Proc. R. Soc. London, Ser. A 208, 108 (1951).

${ }^{16}$ J. Callaway, Phys. Rev. 113, 1046 (1959).

${ }^{17}$ C. J. Glassbrenner and G. A. Slack, Phys. Rev. 134, A1058 (1964).

${ }^{18}$ H. J. McSkimin and P. Andreatch, Jr., J. Appl. Phys. 34, 651 (1963).

${ }^{19}$ P. Flubacher, A. J. Leadbetter, and J. A. Morrison, Philos. Mag. 4, 273 (1959).

${ }^{20}$ B. L. Zink, R. Pietri, and F. Hellman, Phys. Rev. Lett. 96, 055902 (2006).

${ }^{21}$ T. Yoshikawa, T. Yagi, N. Oka, J. Jia, Y. Yamashita, K. Hattori, Y. Seino, N. Taketoshi, T. Baba, and Y. Shigesato, Appl. Phys. Express 6, 021101 (2013).

${ }^{22}$ N. Kim, B. Domercq, S. Yoo, A. Christensen, B. Kippelen, and S. Graham, Appl. Phys. Lett. 87, 241908 (2005). 